\title{
Four-dimensional trajectory optimization model with priority in continuous time period
}

\author{
Meng $\mathrm{Li}^{1}$, Jianyu $\mathrm{Chu}^{2}$, Yinfeng $\mathrm{Li}^{3}$, Xianjie $\mathrm{Wu}^{4}$ \\ ${ }^{1,2,3}$ College of Civil and Architectural Engineering, North China University of Science and Technology, \\ Tangshan, China \\ ${ }^{3}$ State Key Laboratory of Air Traffic Management System and Technology, CETC 28, Nanjing, China \\ ${ }^{4}$ China Civil Aviation Air Traffic Management Bureau in North China, Beijing, China \\ ${ }^{2}$ Corresponding author \\ E-mail:1472698360@qq.com,2jychu@ncst.edu.cn,3ili070320217@126.com,,4michaelwooder@163.com
}

Received 14 May 2021; received in revised form 17 June 2021; accepted 15 July 2021

DOI https://doi.org/10.21595/jmeacs.2021.22056

Check for updates

Copyright (C) 2021 Meng Li, et al. This is an open access article distributed under the Creative Commons Attribution License, which permits unrestricted use, distribution, and reproduction in any medium, provided the original work is properly cited.

\begin{abstract}
This article aims to mitigate the imbalance of capacity and flow in airspace. Targeting to minimize the total delay time, taking the real-life sector operation and aircraft flight rules into consideration, with constraints of sector capacity limit and minimal time interval, the principle to prioritize aircraft when the delay occurred was raised. A trajectory planning model with the priority of aircraft was then established in the model of trajectory based on operation in a continuous period. The traditional genetic algorithm was also improved through the strategy of enhanced elitism preserving and double-stranded chromosome structure. Case studies indicated that the proposed trajectory planning model and solution algorithm have contributed to, over the two periods, an average reduction of $71.77 \%$ in the delay time on optimization effect, and an increase of $19.48 \%$ in the calculation speed. In this case, the model appears to, in a relatively short time, provide a trajectory allocation strategy with security and timeliness, for aircraft operated in consecutive periods. As a result, the sectors can operate without any conflicts while effectively reducing flight delays, minimizing the traffic congestion and potential accidents, so as to take the most advantages of sector resources allocation.
\end{abstract}

Keywords: the priority of aircraft, four-dimensional trajectory, trajectory planning, dualchromosome genetic algorithm.

\section{Introduction}

In recent years, the global air transportation industry has rapidly developed, and the general public's travel demand for air transportation has been steadily increasing. According to the annual report of Air Transport Council published by International Civil Aviation Organization, since 2010, the number of passengers transported worldwide has increased from 2708 million to 4486 million, with an average growth rate of $6.07 \%$ in the past decade. Air transportation has become an important mode for people to travel. While the aviation industry is booming, severe challenges were brought to the current air traffic control system. The contradiction between the limited airspace resources and the ever-increasing flight demands has become one of the main factors restricting the development of the international air transport industry. To address this problem, the international civil aviation community started to actively explore the next generation of air traffic management systems. Trajectory Based on Operation (TBO) is one of the core concepts, which shares dynamic information among air traffic control units, airlines, and aircraft, with coordination between flight and control on decision-making process. TBO is based on the Four-Dimensional Trajectory (4DT) planning of aircraft, which refers to an ordered set of fourdimensional space coordinates (longitude, latitude, height and time) of all sequential points experienced by the aircraft from take-off to landing [1].

Modeling is an important method in natural science research. By abstracting complex problems or structures, analyzing models and implementing appropriate methods, more optimal solutions to the questions can be obtained [2-5]. This research method has also been widely used 
in air traffic management. Trajectory planning is an important technique for allocating airspace resources to ensure the effectiveness of flow management. In order to optimize trajectory planning, researchers have proposed models from different perspectives. After studying marketing principles and time slot dependencies of different airports, Castelli et al. proposed two separate time slot allocation mechanisms to reduce losses due to delays and improve the operation efficiency of air traffic control system [6], [7]. Corolli et al. proposed a stochastic planning model for the allocation of time slots in the airport network when the capacity is randomly reduced [8], with delay being an important indicator [9]. Ivanov et al. designed a two-level mixed integer optimization model for the imbalance of the capacity and flow of the flight, combined with the delay issue of the flow control releases [10]. Soler et al. studied the 4D trajectory planning problem in a sensitive environment of trajectory conversion, then established a mixed-integer optimal control problem model [11]. Zhang proposed a CDM GDP time slot allocation method based on balanced effectiveness, efficiency and fairness, and established a multi-objective optimization model with the goal of minimizing delay and airline loss deviation [12]. Zhang modeled the air traffic network flow optimization problem for four-dimensional trajectory operation to simultaneously optimizes flight cost and fairness [13]. Yang et al. established a risk decision-making model based on the uncertainty of flight operation time to minimize flight delays while ensuring the stability of route operations [14]. Zhang et al. aimed at solving the problem of multi-airspace trajectory planning in the pre-tactical stage, and achieved the goal of generating conflict-free trajectories by changing the time and height of key points [15]. Yang proposed the trajectory planning method in the pre-tactical stage, and established the pre-tactical aircraft trajectory planning theory based on the centralized processing of flight plans [16]. With air route operation as the object, Chen et al investigated on autonomous trajectory planning and conflict management technology for restricted airspace [17]. In terms of model algorithm research, $\mathrm{Wu}$ proposed an airport arrival and departure slot allocation model based on emergencies, and introduced a biogeographic algorithm [18]. Xu et al. targeted on minimizing the total flight delay costs, established a comprehensive management decision model for ground waiting and trailing interval, and used a hybrid coding genetic algorithm in the model [19]. Li et al. proposed an improved $\mathrm{A}^{*}$ search algorithm for the multi-constrained aircraft trajectory planning problem expressed in grid form [20].

In terms of trajectory planning, existing research was mainly aimed at microscopic trajectory planning for a certain air route or airspace unit such as an airport. By establishing an optimization model, the transit time and route are planned for the aircraft. In order to meet the flight requirements of the airspace, while minimizing conflicts between capacity and flow in the sector, this article sets up a 4DT planning model prioritizing aircraft for a continuous period, and designs a diploid genetic algorithm with an elitism strategy to solve the problem.

\section{Trajectory planning model}

From a macro perspective, aiming at mitigating the imbalance of capacity and flow in the sector, considering the delay spread, a 4DT planning model with priority of aircraft is established, which plans the sector entry time for the aircraft in two consecutive time periods. As a result, the number of conflicts between aircraft can be reduced and the balance of capacity and flow in the sector can be achieved.

\subsection{Modeling ideas}

Airspace and airport capacity refers to the maximum number of aircraft sorties that an airspace and airport can receive in a specific period of time. The waypoints, routes and their capacity in the airspace constitute a weighted air traffic network directed graph, which thoroughly reflects the airspace operation status and restriction information. As shown in Fig. 1, $P$ is the waypoint; $R$ is the route; $f$ is the flow; and $c$ is the capacity. The conflict of capacity and flow in busy airspace 
sectors not only causes congestion in the air traffic network, but exposes a significant threat to the safety of the traffic flow. Aiming at resolving the airspace congestion problem caused by excessive traffic in the sector, this article takes the shortest entry delay as the optimization goal from a macro perspective, while constraints include the restrictions between capacity and flow, the minimum time interval between aircraft, along with the priority of the aircraft. Consequently, a 4DT planning model for continuous-period with the priority of aircraft is established, which is to program the sector entry time in continuous-period and reach the balance between capacity and flow in the airspace.

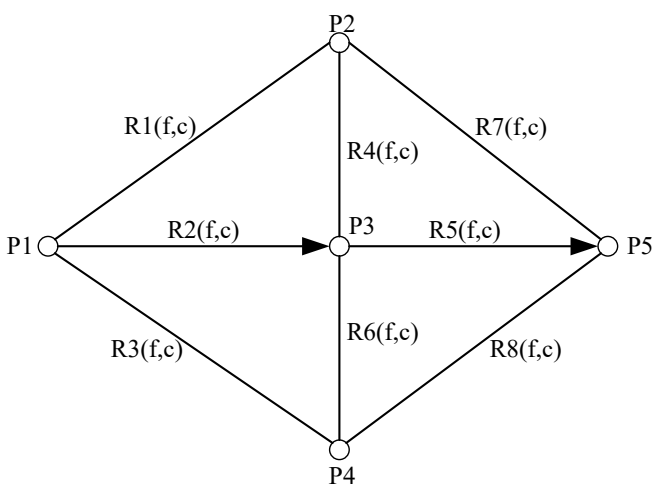

Fig. 1. Schematic diagram of air traffic network

\subsection{Basic assumptions}

In order to describe the problem concisely and comprehensively, the following assumptions are established:

(1) Treat the aircraft as a mass point;

(2) Each aircraft enters the sector at the scheduled time;

(3) Both upstream and downstream sectors are in a state of balance between capacity and flow;

(4) The existing aircraft in the sector are not considered.

\subsection{Model parameters}

The symbol parameters used in the model are defined as shown in Table 1.

Table 1. Symbol definitions in the model

\begin{tabular}{|c|c|}
\hline Symbol & Definition \\
\hline$S$ & Sector \\
\hline$f_{T_{1}}=\left\{f_{1}, f_{2}, \ldots, f_{n}\right\}$ & $\begin{array}{r}\text { The flow of aircrafts entering the sector in } T_{1} \text { time period, } \\
n \text { refers to the total number of aircraft }\end{array}$ \\
\hline$f_{T_{2}}=\left\{f_{1}, f_{2}, \ldots, f_{k}\right\}$ & $\begin{array}{r}\text { The flow of aircrafts entering the sector in } T_{2} \text { time period, } \\
k \text { refers to the total number of aircraft }\end{array}$ \\
\hline$C_{S}\left(T_{i}\right)$ & The capacity of the sector in $T_{i}$ time period, $i=1,2$ \\
\hline$Q_{S}\left(T_{i}\right)$ & The scheduled flow of the sector in $T_{i}$ time period, $i=1,2$ \\
\hline$Q_{S}^{\prime}\left(T_{i}\right)$ & The planned flow of the sector in $T_{i}$ time period, $i=1,2$ \\
\hline$P_{a k\left(f_{m}\right)}$ & The aircraft $f_{m}$ in the entry point $P_{a k}$ \\
\hline$\varphi$ & The minimum time interval, min \\
\hline$t_{a a}$ & Scheduled entry time of aircraft $f_{a}$ \\
\hline$t_{a a}^{\prime}$ & Planned entry time of aircraft $f_{a}$ \\
\hline$f_{a\left(P_{a k}\right)}$ & The entry point of aircraft $f_{a}$ is $P_{a k}$ \\
\hline$f_{x}=\left\{f_{1}, f_{2}, \ldots, f_{\lambda}\right\}$ & After $T_{1}$ time period, the collection of aircraft $f_{x}$ \\
& which $t_{a a}^{\prime} \in T_{2}, \lambda$ refers to the number of those aircraft \\
\hline
\end{tabular}




\subsection{Optimization model}

The objective function and constraint conditions of the model are shown in Eqs. (1-5):

$f=\min \sum_{a=1}^{n}\left(t_{a a}^{\prime}-t_{a a}\right)$,

s.t. $\mu \cdot C_{s}\left(T_{i}\right) \leq Q_{s}^{\prime}\left(T_{i}\right) \leq C_{s}\left(T_{i}\right)$,

$\left|t_{a\left(P_{a k}\left(f_{m+1}\right)\right.}^{\prime}-t_{a\left(P_{a k} f_{m)}\right)}^{\prime}\right| \geq \varphi$,

$t_{a a}^{\prime} \geq t_{a a}$

$t_{a\left(P_{a k}\left(f_{\lambda}\right)\right.}^{\prime}>t_{a\left(P_{a k(f a)}\right)}^{\prime}$

Eq. (1) is the objective function, which indicates that the total delay of arrival of all aircraft in the two time periods is the smallest; Eq. (2) represents the balance constraint between capacity and flow, that is, within a time period, the flow in sector $S$ must be less than or equal to the capacity, and greater than or equal to $\mu$ times the capacity ( $\mu$ is a constant, $\mu<1$ ), in order to ensure the flight safety of aircraft while not wasting airspace resources; Eq. (3) is the time interval constraint for the entry time, that is, for aircraft with the same entry waypoints, the sector entry time must meet the minimum time interval to avoid conflicts between aircraft; Eq. (4) means that the constraint of not arrived in advance, that is, the planned sector entry time of the aircraft cannot be earlier than the scheduled time; Eq. (5) is the flight priority constraint, that is, after the planning in the first stage $T_{1}$, the aircraft, whose entry time $t_{a a}^{\prime}$ are within the second time period $T_{2}$, which means the planned time must be earlier than the other flights with the same entry waypoint.

\subsection{Model solving}

Due to the complex problems and constraints of the model in this article, a genetic algorithm with better search ability and convergence performance is selected to be implemented in the model. Genetic algorithm is a bionic algorithm for searching the optimal solution conceived based on the principle of biological evolution. It simulates the natural process of gene recombination and evolution, converts the parameters of the problem into genes through coding. Several genes form a chromosome, which is treated as an individual. A number of chromosomes experience natural selection, paired crossover and mutation. After multiple iterations, the most optimized result is obtained. In order to improve the optimization ability and reduce the runtime of the algorithm, this article improves the genetic algorithm and designs a dual-chromosome genetic algorithm with strategy of enhanced elitism preserving. The algorithm steps are shown in Fig. 2.

\subsubsection{Encoding}

Considering the complex constraints of the problem, this article chooses the real-valued encoding, calculates the scheduled entry time of each aircraft as the time difference compared to 00:00:00 of the day and converts it into minutes. This real value is expressed as a gene. In addition, the number of decision variables in the problem is large, so dual-stranded chromosomes that are more in line with diploid biological characteristics are designed, so that the decision variables are evenly distributed on two chromosomes, which are expressed as an individual. For example, there are 10 aircraft in the question, and their scheduled entry time are 08:00:00, 08:06:00, 08:10:00, 08:15:00, 08:04:00, 08:10:00, 08:16:00, 08:25:00, 08:20:00, 08:14:00, the dual-chromosome genotypes with real value encoding are shown in Table 2 .

Table 2. Dual-chromosome structure with real-valued encoding

\begin{tabular}{|l|l|l|l|l|l|}
\hline $\mathrm{X} 1$ & 480 & 486 & 490 & 495 & 484 \\
\hline $\mathrm{X} 2$ & 490 & 496 & 500 & 505 & 494 \\
\hline
\end{tabular}




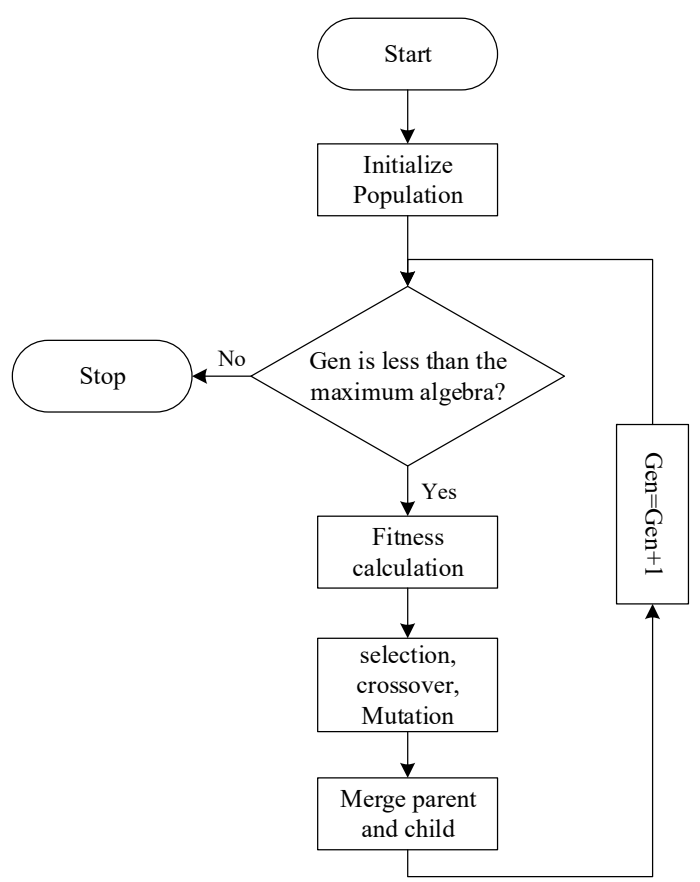

Fig. 2. Genetic algorithm steps

\subsubsection{Genetic operation}

In this article, the strategy of enhanced elite retention is added to the dual-chromosome genetic algorithm; moreover, the tournament selection operator, the two-point crossover operator and the breeder genetic algorithm mutation operator are used.

Specifically, the elite selection strategy is the optimal preservation strategy, which means that the most adaptive individual in the current population does not participate in crossover and mutation, but directly replaces the least after genetic manipulation. The elite selection strategy is a part of the selection operation, which ensures that the currently obtained optimal individual will not be destroyed by the genetic operation and make the genetic algorithm converge globally. The tournament selection operator refers to taking a certain number of individuals from the population each time, then selecting the best individual to enter the offspring, and repeating this operation until the required population size is reached. Additionally, the two-point crossover operator randomly sets two crossover points in the individual code string, then performs partial gene exchange. Finally, the breeder genetic algorithm mutation operator is a population matrix for real-valued coding, and the mutation distance can be controlled by the available compression rate and gradient division. The smaller the compression rate and the more gradient divisions, the smaller the range of variation and the stronger the local search capability.

\section{Case analysis}

\subsection{Algorithm running results}

According to the historical flight data of North China in October 2018, the traffic flow of each sector was analyzed in the busy sector and period, and the data of the Beijing ACC05 sector on October 28, 2018, 16:00:00-17:00:00 was selected as the basis. The process of data preprocessing is shown in Table 3.

The selected period was divided into two consecutive periods, and the entry time of the aircraft which will enter the sector is planned. Specifically, 16:00:00-16:29:59 is the first time period $T_{1}$, 
and 16:30:00-17:00:00 is the second time period $T_{2}$. The sector structure of Beijing ACC05 is shown in Fig. 3: there are 7 important routes and 3 main entry points in the sector, named ISGOD, LARAD-B458-UBTAB, and ENGIL. According to the traffic capacity table in North China, the ACC05 sector has a half-hour capacity of 23 sorties. This article uses Python, combined with the third-party genetic algorithm toolbox Geatpy to programming the algorithm.

Table 3. The process of data preprocessing

\begin{tabular}{|c|c|}
\hline Step 1 & $\begin{array}{r}\text { Data filtering. In the historical flight plan data table, } \\
\text { all the flight information passing through the ACC05 sector was filtered out }\end{array}$ \\
\hline Step 2 & $\begin{array}{c}\text { Data cleaning. Flights that have the same entry and leave time were excluded. } \\
\text { Such data was unavailable due to control reasons, pilot operations or system reasons }\end{array}$ \\
\hline Step 3 & $\begin{array}{r}\text { Calculating the traffic of the ACC05 sector for each hour, and the data } \\
\text { of the busy period where there is a risk of conflict between capacity } \\
\text { and flow was selected, which was 16:00:00-17:00:00 }\end{array}$ \\
\hline Step 4 & Based on the original route, confirming the entry waypoint of the aircraft in the selected period \\
\hline
\end{tabular}

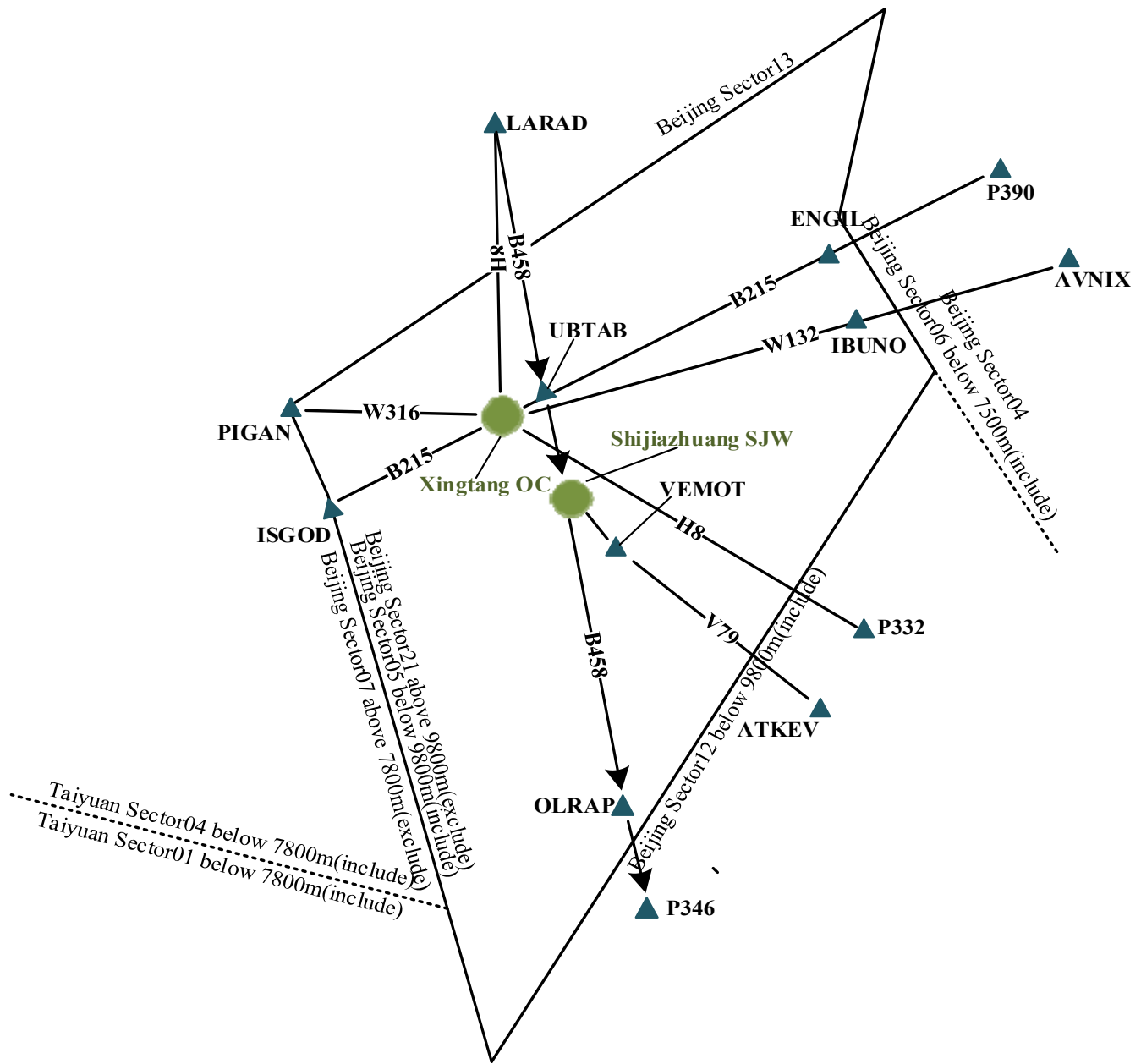

Fig. 3. ACC05 sector structure diagram

In the genetic algorithm, the population size is set to 60 with the maximum evolutionary generation being 300. According to the optimization model and the improved genetic algorithm proposed in the article, the sector entry time of aircraft in a continuous period is planned. The final 
entry time optimization strategy and delay time of each aircraft are shown in Table 4.

Table 4. The entry time optimization strategy

\begin{tabular}{|c|c|c|c|c|c|c|c|}
\hline \multicolumn{4}{|c|}{$T_{1}$ time period } & \multicolumn{4}{|c|}{$T_{2}$ time period } \\
\hline Sum & \multicolumn{3}{|c|}{25 sorties } & Sum & \multicolumn{3}{|c|}{27 sorties } \\
\hline Flight & $\begin{array}{c}\text { Scheduled entry } \\
\text { time }\end{array}$ & $\begin{array}{l}\text { Planned } \\
\text { entry time }\end{array}$ & $\begin{array}{c}\text { Delay } \\
\text { time }\end{array}$ & Flight & $\begin{array}{l}\text { Scheduled } \\
\text { entry time }\end{array}$ & $\begin{array}{c}\text { Planned } \\
\text { entry time }\end{array}$ & \begin{tabular}{|c} 
Delay \\
time
\end{tabular} \\
\hline HBH3275 & $16: 08: 00$ & $16: 08: 00$ & 0 & CES2111 & $16: 55: 00$ & $16: 55: 00$ & 0 \\
\hline CQH8641 & $16: 20: 00$ & $16: 20: 00$ & 0 & HBH3217 & $16: 59: 00$ & $\begin{array}{ll}16: 59: 00 \\
\end{array}$ & 0 \\
\hline CQH8927 & $16: 28: 00$ & $16: 28: 00$ & 0 & CSC8103 & $16: 44: 00$ & $17: 00: 00$ & 16 \\
\hline CHH7238 & $16: 27: 00$ & $16: 30: 00$ & 3 & CQH8661 & $16: 39: 00$ & $16: 39: 00$ & 0 \\
\hline CSN635 & $16: 14: 00$ & $16: 14: 00$ & 0 & CHH7229 & 16:53:00 & 16:53:00 & 0 \\
\hline CSN6362 & $16: 25: 00$ & $16: 30: 00$ & 5 & CSN6275 & $16: 33: 00$ & $17: 02: 00$ & 29 \\
\hline CSN6482 & $16: 16: 00$ & $16: 17: 00$ & 1 & CSN6309 & $16: 49: 00$ & $17: 08: 00$ & 19 \\
\hline HBH3278 & $16: 15: 00$ & $16: 15: 00$ & 0 & CES5707 & $16: 46: 00$ & $16: 46: 00$ & 0 \\
\hline CQH8641 & $16: 22: 00$ & $16: 22: 00$ & 0 & CCA1224 & $16: 35: 00$ & $16: 35: 00$ & 0 \\
\hline CCA4143 & $16: 27: 00$ & $16: 27: 00$ & 0 & CQH8661 & $16: 43: 00$ & $16: 43: 00$ & 0 \\
\hline CCA8208 & $16: 11: 00$ & $16: 11: 00$ & 0 & CCA4109 & $16: 34: 00$ & $16: 38: 00$ & 4 \\
\hline CHH7782 & $16: 24: 00$ & $16: 29: 00$ & 5 & LKE9728 & $16: 31: 00$ & $16: 31: 00$ & 0 \\
\hline CHH7801 & 16:18:00 & $16: 23: 00$ & 5 & CHH7229 & $16: 57: 00$ & $16: 57: 00$ & 0 \\
\hline CES5527 & $16: 20: 00$ & $16: 20: 00$ & 0 & CSN3602 & $16: 38: 00$ & $16: 56: 00$ & 18 \\
\hline CCA1365 & $16: 26: 00$ & $16: 26: 00$ & 0 & CXA8239 & $16: 42: 00$ & $16: 44: 00$ & 2 \\
\hline CHH7329 & $16: 21: 00$ & $16: 23: 00$ & 2 & HXA2792 & $16: 51: 00$ & $17: 06: 00$ & 15 \\
\hline CHH7590 & 16:03:00 & $16: 10: 00$ & 7 & CES781 & $16: 39: 00$ & $16: 39: 00$ & 0 \\
\hline CHH7264 & 16:16:00 & $16: 16: 00$ & 0 & CSC8809 & $16: 41: 00$ & $16: 41: 00$ & 0 \\
\hline HXA2669 & $16: 29: 00$ & $16: 29: 00$ & 0 & CCA871 & $16: 46: 00$ & $16: 46: 00$ & 0 \\
\hline CHH7879 & $16: 10: 00$ & $16: 20: 00$ & 10 & SNG4310 & $16: 55: 00$ & $17: 03: 00$ & 8 \\
\hline CSN6365 & 16:07:00 & $16: 07: 00$ & 0 & AMU001 & $16: 32: 00$ & $16: 36: 00$ & 4 \\
\hline CES2748 & $16: 03: 00$ & $16: 03: 00$ & 0 & CSN6165 & $16: 53: 00$ & $17: 11: 00$ & 18 \\
\hline CSN3734 & $16: 25: 00$ & $16: 33: 00$ & 8 & CCA1875 & $16: 55: 00$ & $17: 05: 00$ & 10 \\
\hline CSN3138 & $16: 00: 00$ & $16: 00: 00$ & 0 & CSN323 & $16: 53: 00$ & $16: 53: 00$ & 0 \\
\hline CHH7264 & $16: 17: 00$ & $16: 19: 00$ & 2 & CUA5807 & $16: 55: 00$ & $16: 59: 00$ & 4 \\
\hline 1 & 1 & & & CHH7080 & $16: 34: 00$ & $16: 41: 00$ & 7 \\
\hline 1 & 1 & & & CGZ7121 & $16: 50: 00$ & $16: 50: 00$ & 0 \\
\hline
\end{tabular}

In Table 4 , there are 25 aircraft in the $T_{1}$ time period. After planning the entry time of the aircraft that enters the sector in the first time period, the entry time of the aircraft $\mathrm{CHH} 7238$, CSN6362, CSN3734 delays into the second time period. According to the principle to prioritize aircraft when the delay occurred, the entry time of these three aircraft must be earlier than other aircraft with the same entry way points in $T_{2}$ time period, to satisfy the timeliness requirements of aircraft operations. The optimization process of the entry time of the three aircraft with delays under the priority strategy is shown in the Table 5 . As can be seen in Table 5, after the optimization of the priority strategy, the overall delay of the three flights has been reduced by $20 \%$.

Table 5. The optimization process under the priority strategy

\begin{tabular}{|c|c|c|c|}
\hline Flight & Scheduled entry time & Planned entry time after $T_{1}$ & Planned entry time after $T_{2}$ \\
\hline CHH7238 & $16: 27: 00$ & $16: 30: 00$ & $16: 30: 00$ \\
\hline CSN6362 & $16: 25: 00$ & $16: 35: 00$ & $16: 30: 00$ \\
\hline CSN3734 & $16: 25: 00$ & $16: 32: 00$ & $16: 33: 00$ \\
\hline
\end{tabular}

The evolution results of genetic algorithm in the first time period $T_{1}$ and the second time period $T_{2}$ are shown in Fig. 4(a) and Fig. 4(b) respectively. 


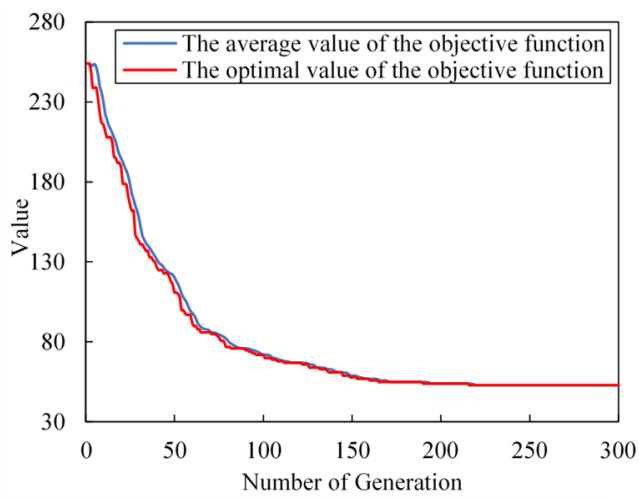

a) Results in $T_{1}$

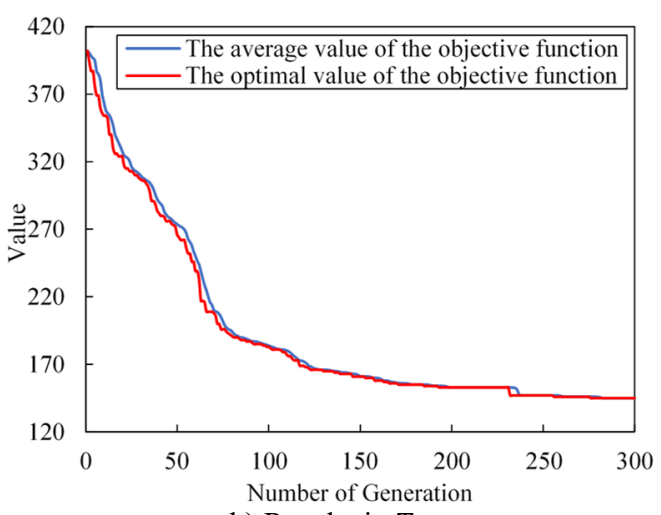

b) Results in $T_{2}$

Fig. 4. Algorithm evolution results

It can be seen from Fig. 4(a) and Fig. 4(b) that the dual-chromosome genetic algorithm with enhanced elite retention strategy adopted in this article is stable in convergence. During the evolution of the solution in $T_{1}$ time period and the $T_{2}$ time period, although the optimal and average values of the individual objective function values of the population fluctuate, they have similar trends and generally show a downward trend, and both curves stabilized around the 230th generation. At this time, the optimal objective function value for the $T_{1}$ time period is 52 , and the optimal objective function value for the $T_{2}$ time period is 157 .

\subsection{Algorithm comparison}

In order to verify the optimization effect and the runtime of the algorithm, the algorithm is compared with the simple genetic algorithm. In the simple genetic algorithm, roulette wheel selection, partial matching crossover, and reverse transformation are used, with the population size is set to 60; the maximum evolutionary generation set to 300 . The results are shown in Table 6 .

Table 6. Comparison between the algorithm in this paper and the simple genetic algorithm

\begin{tabular}{|c|c|c|c|c|}
\hline \multirow{2}{*}{} & \multicolumn{2}{|c|}{$T_{1}$ time period } & \multicolumn{2}{c|}{$T_{2}$ time period } \\
\cline { 2 - 5 } & Calculation speed / & Delay time / min & Calculation speed / & Delay time / min \\
\hline $\begin{array}{c}\text { The algorithm of this } \\
\text { article }\end{array}$ & 1.86 & 52 & 2.80 & 157 \\
\hline $\begin{array}{c}\text { Simple genetic } \\
\text { algorithm }\end{array}$ & 1.89 & 271 & 4.40 & 445 \\
\hline
\end{tabular}

Table 6 shows that in terms of the optimization effect, the algorithm reduces the delay time by $80.81 \%$ and $62.72 \%$ in the two time periods compared with the simple genetic algorithm. In terms of calculating speed, compared with the simple genetic algorithm, the algorithm in this article was $2.59 \%$ and $36.36 \%$ faster in the two time periods. Due to the limitation of the algorithm itself, the simple genetic algorithm produced a premature phenomenon in the process of solving this problem and fell into a local optimal solution. In summary, the dual-chromosome genetic algorithm with enhanced elite retention strategy adopted in this article has significant advantages in optimization effect and calculation speed. Simultaneously, it can provide an entry time strategy with the highest safety and the shortest delay time in a relatively short period of time, which can be applied to the current industry, in order to mitigate the risk of conflicts between aircraft, and improve the utilization of sector resources.

\section{Conclusions}

Based on historical flight plan data in North China, regarding the conflict between capacity 
and flow, the principle to prioritize aircraft when the delay occurred is raised. This article establishes a 4DT optimization model with priority of aircraft for continuous periods of time, then designs double-stranded chromosome structure and the strategy of enhanced elitism preserving to improve the traditional genetic algorithm. Experimental results illustrate that the trajectory planning model and algorithm proposed are stable and efficient, and can provide both applicability and safety of the entry time allocation strategy in a relatively short period of time. The research in this paper conforms to the real-life operation of air traffic management and can meet the actual needs of controllers. It has guiding significance for the traffic flow management, conflict detection and relief of the busy sector. It laid a research foundation for the follow-up trajectory prediction and planning in TBO mode.

\section{Acknowledgements}

This research was supported by Science and Technology Project of North China Regional Air Traffic Management Bureau CAAC, (No. 201904; 202002) and 2020 Civil Aviation Safety Ability Building Funding Project (202072).

\section{References}

[1] L. Li, "Core concept of the next-generation ATC system: operation based on 4 dimensional trajectory," (in Chinese), China Civil Aviation, Vol. 207, No. 8, pp. 18-20, 2015.

[2] B. Tan, Y. Chen, Q. Liao, B. Zhang, N. Zhang, and Q. Xie, "A condensed dynamic model of a heavyduty truck for optimization of the powertrain mounting system considering the chassis frame flexibility," Proceedings of the Institution of Mechanical Engineers, Part D: Journal of Automobile Engineering, Vol. 234, No. 10-11, pp. 2602-2617, Sep. 2020, https://doi.org/10.1177/0954407020909241

[3] M. Wang, B. Zhang, Y. Chen, N. Zhang, and J. Zhang, "Frequency-based modeling of a vehicle fitted with roll-plane hydraulically interconnected suspension for ride comfort and experimental validation," IEEE Access, Vol. 8, pp. 1091-1104, 2020, https://doi.org/10.1109/access.2019.2935260

[4] M. Zheng, P. Peng, B. Zhang, N. Zhang, L. Wang, and Y. Chen, "A new physical parameter identification method for two-axis on-road vehicles: simulation and experiment," Shock and Vibration, Vol. 2015, pp. 1-9, 2015, https://doi.org/10.1155/2015/191050

[5] H. Qi, Y. Chen, N. Zhang, B. Zhang, D. Wang, and B. Tan, "Improvement of both handling stability and ride comfort of a vehicle via coupled hydraulically interconnected suspension and electronic controlled air spring," Proceedings of the Institution of Mechanical Engineers, Part D: Journal of Automobile Engineering, Vol. 234, No. 2-3, pp. 552-571, Feb. 2020, https://doi.org/10.1177/0954407019856538

[6] L. Castelli, R. Pesenti, and A. Ranieri, "The design of a market mechanism to allocate air traffic flow management slots," Transportation Research Part C: Emerging Technologies, Vol. 19, No. 5, pp. 931-943, Aug. 2011, https://doi.org/10.1016/j.trc.2010.06.003

[7] L. Castelli, P. Pellegrini, and R. Pesenti, "Airport slot allocation in Europe: economic efficiency and fairness," International Journal of Revenue Management (IJRM), Vol. 6, No. 1/2, pp. 28-44, 2012.

[8] L. Corolli, G. Lulli, and L. Ntaimo, "The time slot allocation problem under uncertain capacity," Transportation Research Part C: Emerging Technologies, Vol. 46, pp. 16-29, Sep. 2014, https://doi.org/10.1016/j.trc.2014.05.004

[9] J. Zhang, B. Zhang, N. Zhang, C. Wang, and Y. Chen, "A novel robust event-triggered fault tolerant automatic steering control approach of autonomous land vehicles under in-vehicle network delay," International Journal of Robust and Nonlinear Control, Vol. 31, No. 7, pp. 2436-2464, May 2021, https://doi.org/10.1002/rnc.5393

[10] N. Ivanov, F. Netjasov, R. Jovanović, S. Starita, and A. Strauss, "Air traffic flow management slot allocation to minimize propagated delay and improve airport slot adherence," Transportation Research Part A: Policy and Practice, Vol. 95, pp. 183-197, Jan. 2017, https://doi.org/10.1016/j.tra.2016.11.010

[11] M. Soler, B. Zou, and M. Hansen, "Flight trajectory design in the presence of contrails: Application of a multiphase mixed-integer optimal control approach," Transportation Research Part C: Emerging Technologies, Vol. 48, pp. 172-194, Nov. 2014, https://doi.org/10.1016/j.trc.2014.08.009 
[12] M. H. H. H. H. Zhang, "Multi-objection optimization allocation of aircraft landing slot in CDM GDP," (in Chinese), Journal of Systems and Management, Vol. 18, No. 3, pp. 302-308, 2009.

[13] X. Zhang, "Research on air traffic network flow optimization problem for four-dimensional trajectory," M.A. Thesis, Beihang University, Beijing, 2014.

[14] S. W. Yang, P. Chen, and M. Tong, "A model of en-route and slot allocation based on uncertainty of flight time," (in Chinese), Journal of Transport Information and Safety, Vol. 37, No. 6, pp. 156-162, 2019.

[15] Y. Zhang, Y. B. Ding, and Y. J. Yang, "A multi-airspace trajectory planning and negotiation method for four-dimensional trajectory operation," China Pantent, CN201811207110.X, 2019.

[16] D. He, "Research on aircraft trajectory planning based on flight plan centralized processing," M.A. Thesis, Civil Aviation Flight University of China, Guanghan, Sichuang Province, China, 2020.

[17] Y. T. Chen et al., "Autonomous trajectory planning and conflict management technology in restricted airspace," (in Chinese), Acta Aeronautica et Astronautica Sinica, Vol. 41, No. 9, p. 324045, 2020.

[18] D. H. Wu, "Research on optimization techniques of airport slot allocation," M.A. Thesis, Nanjing University of Aeronautics and Astronautics, Nanjing, Jiangsu Province, China, 2016.

[19] D. H. Xu, Y. Zhang, and P. Chen, "Research on ground delay and miles-in-trail composite decision making models and algorithms," (in Chinese), Aeronautical Computing Technique, Vol. 49, No. 1, pp. 54-58, 2019.

[20] S. B. Li, X. X. Su, and H. J. Li, "Realization of 2D grid-based route planning algorithm," (in Chinese), Electronics Optics and Control, Vol. 14, No. 2, pp. 34-37, 2007.

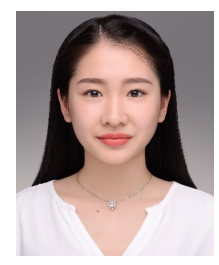

Meng Li received the Bachelor degree in engineering management from North China University of Science and Technology, Tangshan, China, in 2018, where she is now pursuing the Master degree. Her current research interests include air traffic management and track planning techniques.

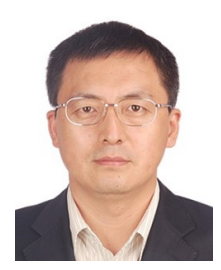

Prof. Jianyu Chu received the $\mathrm{Ph}$. D. degree in civil engineering from Tianjin University, China, in 2015. He is currently a full Professor in North China University of Science and Technology. His research interests include emergency traffic planning and comprehensive disaster prevention planning.

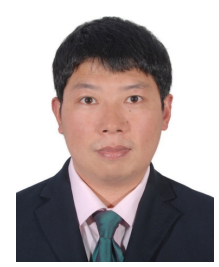

Dr. Yinfeng li received the B.S. and Ph.D. degrees from Nanjing University of Aeronautics and Astronautics, Nanjing, China, in 2007 and 2015, respectively. He is currently an Associate Professor in the College of Civil and Architectural Engineering, North China University of Science and Technology. His research interests include air traffic flow management, airspace management and traffic data analysis.

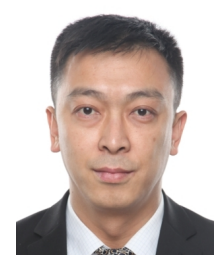

Xianjie Wu graduated from Civil Aviation University of China. He joined North China ATMB of CAAC in 2003 and he is currently the director of Flight Plan Management Department of Operation Management Center. His research interests include air traffic operation management, air traffic flow management and data analysis. 